\title{
Measuring hemoglobin spectra: searching for carbamino-hemoglobin
}

\author{
Emmanuel Dervieux $\odot$, a,b,* Quentin Bodinier $\odot,{ }^{\text {a }}$ Wilfried Uhring $\odot,{ }^{b}$ \\ and Michaël Théron ${ }^{c}$ \\ ${ }^{a} B i O S E N C Y$, Cesson-Sévigné, France \\ ${ }^{b}$ University of Strasbourg and CNRS, Strasbourg Cedex, France \\ ${ }^{c}$ Université de Bretagne Occidentale, ORPHY, Brest, France
}

\begin{abstract}
Significance: The arterial carbon dioxide $\left(\mathrm{CO}_{2}\right)$ partial pressure $\mathrm{Pa}_{\mathrm{CO}_{2}}$ is a clinically relevant variable. However, its measurement requires arterial blood sampling or bulky and expensive transcutaneous $\mathrm{Ptc}_{\mathrm{CO}_{2}}$ meters. While the spectrophotometric determination of hemoglobin species-such as oxy-hemoglobin $\left(\mathrm{O}_{2} \mathrm{Hb}\right)$ and deoxy-hemoglobin $(\mathrm{HHb})$ - allowed for the development of pulse oximetry, the measurement of $\mathrm{CO}_{2}$ blood content with minimal discomfort has not been addressed yet.
\end{abstract}

Aim: Characterizing human carbamino-hemoglobin $\left(\mathrm{CO}_{2} \mathrm{Hb}\right)$ absorption spectrum, which is missing from the literature. Providing the theoretical background that will allow for transcutaneous, noninvasive $\mathrm{Pa}_{\mathrm{CO}_{2}}$ measurements.

Approach: A tonometry-based approach was used to obtain gas-equilibrated, lysed, diluted human blood. Equilibration was performed with both $\mathrm{CO}_{2}$, dinitrogen $\left(\mathrm{N}_{2}\right)$, and ambient air. Spectrophotometric measurements were carried out on the 235- to 1000-nm range. A theoretical background was also derived from that of pulse oximetry.

Results: The absorption spectra of both $\mathrm{CO}_{2} \mathrm{Hb}$ and $\mathrm{HHb}$ were extremely close and comparable with that of state-of-the-art $\mathrm{HHb}$. The above-mentioned theoretical background led to an estimated relative error above $30 \%$ on the measured amount of $\mathrm{CO}_{2} \mathrm{Hb}$ in a subject's blood. Auxiliary measurements revealed that the use of ethylene diamine tetraacetic acid did not interfere with spectrophotometric measurements, whereas sodium metabisulfite did.

Conclusions: $\mathrm{CO}_{2} \mathrm{Hb}$ absorption spectrum was measured for the first time. Such spectrum being close to that of $\mathrm{HHb}$, the use of a theoretical background based on pulse oximetry theory for noninvasive $\mathrm{Pa}_{\mathrm{CO}_{2}}$ measurement seems extremely challenging.

(C) The Authors. Published by SPIE under a Creative Commons Attribution 4.0 Unported License. Distribution or reproduction of this work in whole or in part requires full attribution of the original publication, including its DOI. [DOI: 10.1117/1.JBO.25.10.105001]

Keywords: spectrophotometry; hemoglobin; pulse oximetry; transcutaneous monitoring; photoplethysmography.

Paper 200170RR received Jun. 8, 2020; accepted for publication Sep. 28, 2020; published online Oct. 23, 2020.

\section{Introduction}

The accurate determination of blood gases-namely dioxygen $\left(\mathrm{O}_{2}\right)$ and carbon dioxide $\left(\mathrm{CO}_{2}\right)$ - is of crucial importance in medical care since it gives circulatory as well as ventilatory clues on the state of a patient. ${ }^{1}$ In particular, in case of acute respiratory failure, chronic obstructive pulmonary disease or cystic fibrosis, the monitoring of parameters such as the arterial blood dioxygen saturation $\left(\mathrm{SatO}_{2}\right)$ and $\mathrm{CO}_{2}$ arterial pressure $\left(\mathrm{Pa}_{\mathrm{CO}_{2}}\right)$ can strongly affect patient handling and outcome. ${ }^{2-4}$

Pulse oximetry makes it possible to determine $\mathrm{SatO}_{2}$. It is cheap, noninvasive, accurate, and as such, is widely used in clinical context. ${ }^{5,6}$ At the opposite, $\mathrm{Pa}_{\mathrm{CO}_{2}}$ measurements methods are

*Address all correspondence to Emmanuel Dervieux, emmanuel.dervieux@biosency.com 
far less satisfactory. In a nutshell, they consist of arterial blood sampling, airway capnometry, and transcutaneous capnometry.

Arterial blood sampling is the gold standard for $\mathrm{Pa}_{\mathrm{CO}_{2}}$ measurement. ${ }^{7}$ However, it is invasive, can be both painful and risky, ${ }^{8}$ needs expensive blood gas analyzer, and the blood samples must be promptly analyzed upon collection. ${ }^{9}$ To circumvent these flaws, two capnometry methods were developed. Airway capnometry-also known as capnography—is also invasive since it requires the patient to wear a mask or to undergo endotracheal intubation, and it is not reliable in case of end-tidal partial $\mathrm{CO}_{2}$ pressure $\left(\mathrm{Pet}_{\mathrm{CO}_{2}}\right) / \mathrm{Pa}_{\mathrm{CO}_{2}}$ mismatch caused by an increase in physiologic dead space. ${ }^{10,11}$ Finally, transcutaneous capnometry is derived from the StowSeveringhaus electrode ${ }^{12}$ and has been used for a long time in the clinical practice. ${ }^{13}$ Its drawbacks are mainly the need to heat the skin of the patient between $37^{\circ} \mathrm{C}$ and $44^{\circ} \mathrm{C}$, the need for frequent recalibrations of the sensor, and the high price of transcutaneous $\mathrm{CO}_{2}$ monitors $(\sim 15 \mathrm{k} €)$. Consequently, there is a strong need for an alternative to the existing approaches, so as to provide a cheap, noninvasive, and accurate technique for long-term $\mathrm{Pa}_{\mathrm{CO}_{2}}$ monitoring.

The determination of $\mathrm{SatO}_{2}$ with pulse oximetry is made possible by the spectral differences existing between oxyhemoglobin $\left(\mathrm{O}_{2} \mathrm{Hb}\right)$ and deoxyhemoglobin $(\mathrm{HHb})$, allowing to quantify their proportion in arterial blood. It would be extremely interesting if a similar technique was available to distinguish between carbamino-hemoglobin $\left(\mathrm{CO}_{2} \mathrm{Hb}\right)$ and $\mathrm{HHb}$ based on a difference of absorption between these two compounds. Since an equilibrium exists among blood $\mathrm{pH}$, $\mathrm{CO}_{2} \mathrm{Hb}$ content, bicarbonate concentration, and $\mathrm{Pa}_{\mathrm{CO}_{2}},{ }^{14,15}$ the determination of $\mathrm{CO}_{2} \mathrm{Hb}$ blood content would be a first step toward $\mathrm{Pa}_{\mathrm{CO}_{2}}$ determination.

Under such perspective, the starting point is to analyze the $\mathrm{CO}_{2} \mathrm{Hb}$ absorption spectrum. However-at least to the best of our knowledge-its measurement has never been reported in the literature. Consequently, the present article exposes the measurement and analysis of the $\mathrm{CO}_{2} \mathrm{Hb}$ absorption spectrum. The feasibility of pulse carbametry, the equivalent of pulse oximetry, substituting $\mathrm{CO}_{2} \mathrm{Hb}$ to $\mathrm{O}_{2} \mathrm{Hb}$ are also assessed.

\section{Materials and Methods}

\subsection{Hemoglobin Preparation and Measurement}

Although $\mathrm{CO}_{2} \mathrm{Hb}$ absorption spectrum has yet to be measured, a number of authors were interested in measuring the spectra of other hemoglobin species in the past decades. The most complete work on the topic is undoubtedly that of Zijlstra et al., ${ }^{16}$ which summarized almost a century of research aiming at measuring mainly $\mathrm{O}_{2} \mathrm{Hb}, \mathrm{HHb}$, met-hemoglobin (MetHb), and carboxy-hemoglobin $(\mathrm{COHb})$ spectra. Inspired by their work, the following experimental protocol was elaborated:

- Human blood was diluted at 1:10 or 1:1000 [HEPES $20 \mathrm{mM}$, KCL $150 \mathrm{mM}$, pH 7.20, ethylene diamine tetraacetic acid (EDTA) $0.61 \mathrm{mM}$ for the 1:10 dilution only, to prevent coagulation].

- Blood cells were lysed with ultrasound (Sonicator W-10, Heat Systems Ultrasonics).

- The obtained haemolysates were equilibrated using Eschweiler spherical glass tonometers during at least 30 min with either pure dinitrogen $\left(\mathrm{N}_{2}\right)$ - to obtain $\mathrm{HHb}$-or pure $\mathrm{CO}_{2}$ - to obtain $\mathrm{CO}_{2} \mathrm{Hb}$. Alternatively, they were let in ambient air so as to obtain $\mathrm{O}_{2} \mathrm{Hb}$.

- The equilibrated hemolysates were carefully handled so as not to spoil them with ambient air. Syringes and cuvettes were rinsed three times with the tonometry gas, and the solutions were collected from the tonometers through a septum.

- Equilibrated solutions were poured in airtight quartz cuvettes (CV10Q1400FS, Thorlabs, $10 \mathrm{~mm}$ transmitted path length) placed inside the spectrophotometer (Carry 5000, Agilent Technologies).

- Measurements were performed on the 235- to 600-nm range [hereafter referred to as the ultraviolet/visible (UV-Vis) range] for the 1:1000 dilution and on the 600- to 1000-nm range [visible/infrared (Vis-IR) range] for the 1:10 dilution for $\mathrm{N}_{2}$ - and $\mathrm{CO}_{2}$-equilibrated solutions (235 to $590 \mathrm{~nm}$ and 590 to $1000 \mathrm{~nm}$ for air-equilibrated solutions). 


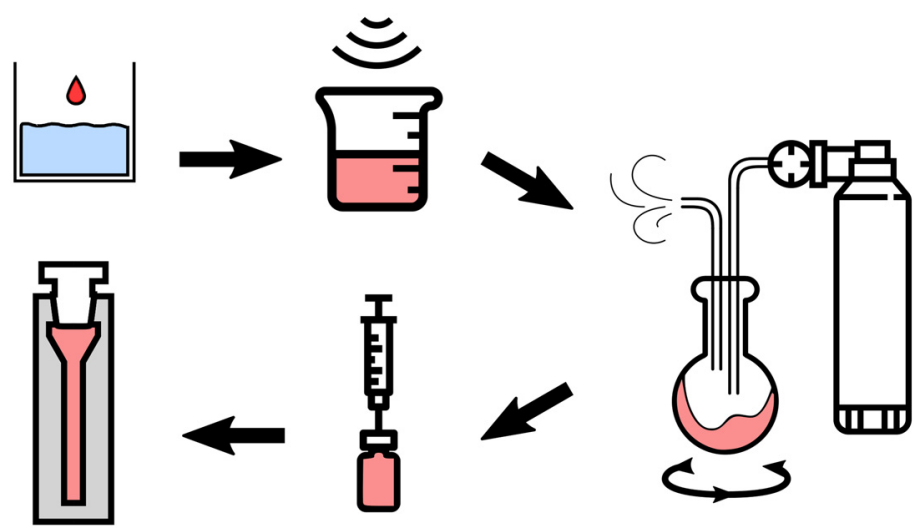

Fig. 1 Preparation of blood, from the vein to the spectrophotometer. The different steps are (from top left to bottom left): venous sampling, dilution, ultrasound lysis, tonometry with $\mathrm{CO}_{2}$ or $\mathrm{N}_{2}$, careful handling, pouring into airtight glass cuvettes for spectrophotometric measurements.

Figure 1 shows the above-described steps, which led to six different mixtures: diluted lysed blood at 1:10 or 1:1000 ratio, equilibrated with $\mathrm{CO}_{2}, \mathrm{~N}_{2}$, or ambient air. In addition, the dilution medium was also measured: pure for baseline correction, and with EDTA and sodium metabisulfite separately, to assess the influence of these substances on the hemolysates measurements. Indeed, no information about the absorption spectrum of sodium metabisulfite nor the one of EDTA in solution is to be found in the literature. An analysis of the relative variations of the spectrum of the dilution medium with and without sodium metabisulfite and EDTA was thus performed. Concentrations in the intramedium were $6.1 \mathrm{mM}$ for EDTA and $2.0 \mathrm{mM}$ for sodium metabisulfite.

In addition, the influence of tonometry duration on the obtained spectra was investigated. Equilibration durations were varied between 30 and $45 \mathrm{~min}$, in $3 \mathrm{~min}$ steps, whereas measuring the absorbance at the 759-nm peak of the spectra of $\mathrm{CO}_{2} \mathrm{Hb}$ and $\mathrm{HHb}$. A Pearson correlation coefficient test was then performed, searching for an influence of the tonometry duration on the measured absorbance values.

\subsection{Assessment of Pulse Carbametry Feasibility}

For pulse carbametry to be achievable, one needs that the absorption spectra of $\mathrm{CO}_{2} \mathrm{Hb}$ and $\mathrm{HHb}$ exhibit sufficient differences at accessible wavelengths. Assessing how much is sufficient is a complex task, which can be answered by addressing the following questions in order:

1. Are the measured average spectra computed for $\mathrm{HHb}$ and $\mathrm{CO}_{2} \mathrm{Hb}$ different?

2. If they are, is this difference statistically significant?

3. Does this difference-observed in the laboratory-translate into something actually exploitable in real life setups, where absorption measurements would be affected by:

- additional absorption caused by tissues surrounding the blood vessels,

- light reflection and scattering in the said tissues,

- intersubject variations in physiology,

- noises related to ambulatory measurement setups (ambient light and motion),

- the accuracy of embedded sensors, which is bound to be lower than that of laboratory spectrophotometers.

Different methods were developed to answer each of these questions, as detailed below.

\subsubsection{Comparison of average spectra for $\mathrm{Hb}$ and $\mathrm{CO}_{2} \mathrm{Hb}$}

After offset correction and outlier removal, a set of spectra for each hemoglobin species was obtained. Each of these sets was then averaged to obtain the final absorption spectrum of the 
corresponding hemoglobin species. Resulting average spectra were then plotted for visual comparison (see Fig. 3).

\subsubsection{Statistical significance of the difference between spectra}

The problem of assessing whether two absorption spectra are statistically different is extremely complex. Indeed, one has to deal with limitations of two kinds:

1. The estimated absorption spectrum of each species is a vector of dependent random variables $\hat{A}_{\text {species }}(\lambda)$ such that $\forall \lambda, \hat{A}_{\text {species }}(\lambda) \sim \mathcal{N}\left[A_{\text {species }}(\lambda), \sigma_{\text {species }}(\lambda)^{2}\right]$ where $\sigma_{\text {species }}(\lambda)$ is the standard variation of the noise on the measurement of the absorption of the species under consideration at wavelength $\lambda$. Thus, deciding whether the difference in the measured spectra of $\mathrm{HHb}$ and $\mathrm{CO}_{2} \mathrm{Hb}$ is statistically significant yields a multivariate analysis problem. Solving the latter would need one to perform at least as many measurements as there are points in the measured spectra (i.e., several hundreds). ${ }^{17}$

2. Variations in the measured spectrum of each species are bound to be induced by the slight differences in the manipulations specific to each species; thus, a statistically significant difference between measured spectra could actually reveal a difference in the protocol and not the spectra themselves.

To address such difficulties, the following analysis was performed on the measured hemoglobin spectra: for each pair of hemoglobin species $\left(\mathrm{HHb}, \mathrm{CO}_{2} \mathrm{Hb}\right)$ and $\left(\mathrm{HHb}, \mathrm{O}_{2} \mathrm{Hb}\right)$, we computed the average, minimum, and maximum relative difference of their absorption spectra. This difference, computed for each wavelength, allows one to assess whether there is an exploitable discrepancy between the spectra of $\mathrm{HHb}$ and $\mathrm{CO}_{2} \mathrm{Hb}$ in the same order of magnitude as that between $\mathrm{HHb}$ and $\mathrm{O}_{2} \mathrm{Hb}$.

\subsubsection{Applicability in real life setups}

To estimate how much difference should be measurable between the spectra of $\mathrm{HHb}$ and $\mathrm{CO}_{2} \mathrm{Hb}$ for it to be exploitable in a pulse carbametry context, parallels were drawn with pulse oximetry. Specifically, we propose to

- use the literature available on pulse oximetry to obtain an estimation of the accuracy of photoplethysmographic measurements on human skin,

- derive a theoretical background for pulse carbametry, and

- use the aforementioned accuracy in such background along with the results of our measurement, to conclude on the feasibility of pulse carbametry.

Note that we focus, in all our analysis, on a two-wavelength system. Even though we acknowledge that multiwavelength approaches can improve the performance of photoplethysmographic systems, the goal of this paper is to evaluate whether there are couples of wavelengths for which the spectra of $\mathrm{HHb}$ and $\mathrm{CO}_{2} \mathrm{Hb}$ exhibit exploitable difference. The optimization of the exploitation of these pairs of wavelengths, should they exist, is out of the scope of this paper.

Accuracy in pulse oximetry. Our goal here is not to dive deeply into the theoretical foundations of pulse oximetry —as it has been done elsewhere ${ }^{18-21}$ — but to estimate the sources of inaccuracy of this technique. In pulse oximetry, $\mathrm{SatO}_{2}$ is derived from the so-called ratio of ratio, $R$, which in turns utilizes absorbance of light by human tissues at two different wavelengths. $R$ is given as

$$
R=\frac{\mathcal{E}_{\mathrm{AC}}\left(\lambda_{1}\right) / \mathcal{E}_{\mathrm{DC}}\left(\lambda_{1}\right)}{\mathcal{E}_{\mathrm{AC}}\left(\lambda_{2}\right) / \mathcal{E}_{\mathrm{DC}}\left(\lambda_{2}\right)}=\frac{\mathcal{E}_{\mathrm{O}_{2} \mathrm{Hb}}\left(\lambda_{1}\right) \cdot \mathrm{SatO}_{2}+\mathcal{E}_{\mathrm{HHb}}\left(\lambda_{1}\right) \cdot\left(1-\mathrm{SatO}_{2}\right)}{\mathcal{E}_{\mathrm{O}_{2} \mathrm{Hb}}\left(\lambda_{2}\right) \cdot \mathrm{SatO}_{2}+\mathcal{E}_{\mathrm{HHb}}\left(\lambda_{2}\right) \cdot\left(1-\mathrm{SatO}_{2}\right)},
$$

where $\lambda_{1}$ and $\lambda_{2}$ are the two measurement wavelengths, often 660 and $940 \mathrm{~nm}$, and $\mathcal{E}$ is either the extinction coefficient of the tissues, measured with photoplethysmography (PPG) and 

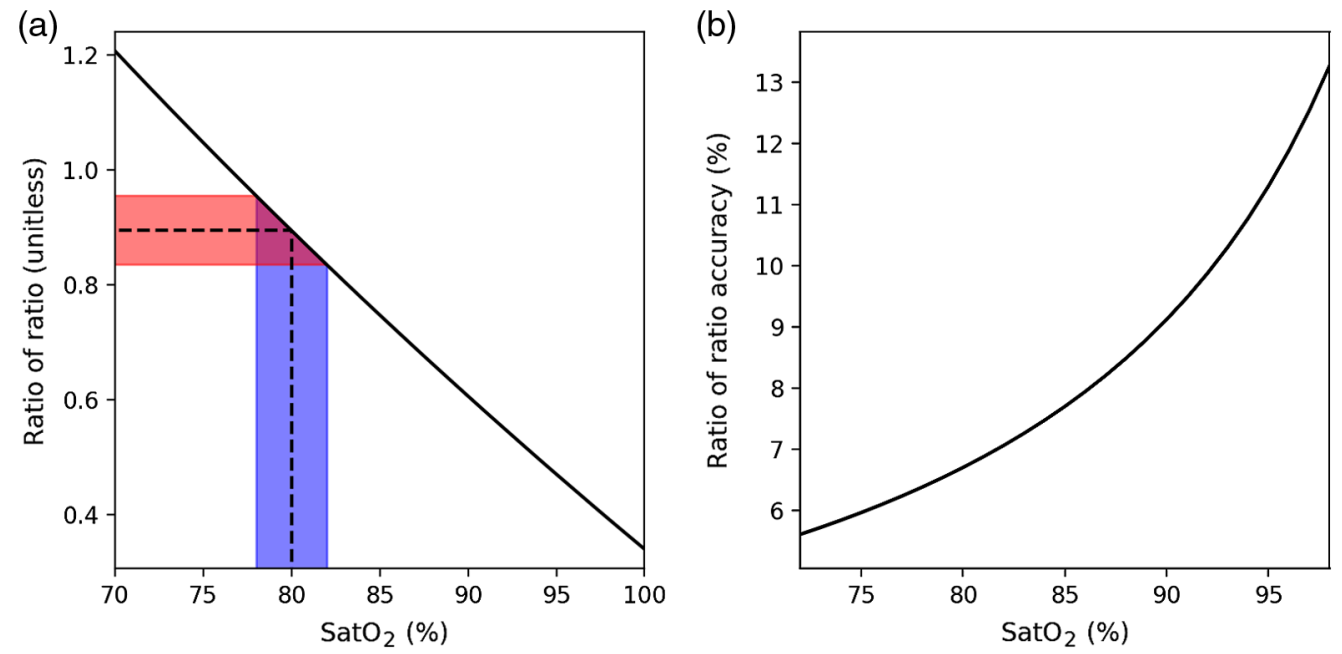

Fig. 2 (a) An error of $2 \%$ on the $\mathrm{SatO}_{2}$ reading at $80 \%$ of $\mathrm{SatO}_{2}$ (in blue) is the result of an inaccuracy of $7 \%$ on the measurement of $R$ (in red). (b) Conversely, if we impose a $2 \%$ error on the $\mathrm{SatO}_{2}$ reading, we can compute the relative variation of $R$, which generated such error.

decomposed in its alternative (AC) and continuous (DC) parts (left hand side) or the extinction coefficient of hemoglobin species (right-hand side).

The question at stake for our analysis is then: what accuracy on $R$ is achievable in practice by common photoplethysmographic sensors? As can be seen from Eq. (1), such an accuracy on $R$ can be estimated based on the accuracy on $\mathrm{SatO}_{2}$ that is reported in the literature. The latter has been studied in many clinical trials - themselves reviewed ${ }^{5,6}$ - and a mean standard deviation of $2 \%$ on the $70 \%$ - to $100 \%-\mathrm{SatO}_{2}$ range can be considered to be achievable under good measurement conditions - that is, mainly no movement from the subject and a good perfusion index. Figure 2 shows the results obtained when taking such a standard deviation for $\mathrm{SatO}_{2}$ and computing the corresponding $R$ measurement inaccuracies at the wavelengths 660 and $940 \mathrm{~nm}$ - the two most-used wavelengths in pulse oximetry-using Zijlstra et al. ${ }^{16}$ absorption coefficient for $\mathrm{O}_{2} \mathrm{Hb}$ and $\mathrm{HHb}$.

One can see that the lower the oxygen saturation is, the more accurate the measurement of $R$ needs to be to guarantee a given accuracy on $\mathrm{SatO}_{2}$. The minimum measured accuracy on $R$ is of $5.6 \%$ at $70 \%$ of $\mathrm{SatO}_{2}$. Such calculations were made with the hypothesis that all errors were Gaussians.

Accuracy in pulse carbametry. Such a minimum error in the case of pulse oximetry gives a best-case-hence optimistic — value of the achievable accuracy on $R$ measurement, using transcutaneous ratiometric techniques. When applying a similar method to obtain the concentration in $\mathrm{CO}_{2} \mathrm{Hb}$ in a pulse carbametry context, one may thus expect-at best-a similar accuracy on $R$. Such a context will be detailed below.

Let us consider a binary system composed solely of $\mathrm{HHb}$ and $\mathrm{CO}_{2} \mathrm{Hb}$ and define carbon dioxide saturation $\left(\mathrm{SatCO}_{2}\right)$ as

$$
\mathrm{SatCO}_{2}=\frac{\mathcal{C}_{\mathrm{CO}_{2} \mathrm{Hb}}}{\mathcal{C}_{\mathrm{CO}_{2} \mathrm{Hb}}+\mathcal{C}_{\mathrm{HHb}}}
$$

where $\mathcal{C}$ is the concentration of the different species involved. Using the pulse oximetry theoretical background, ${ }^{5} \mathrm{SatCO}_{2}$ can be derived from a measured ratio of ratio $R$ as

$$
\operatorname{SatCO}_{2}\left(\lambda_{1}, \lambda_{2}\right)=\frac{\mathcal{E}_{\mathrm{HHb}}\left(\lambda_{1}\right)-R \cdot \mathcal{E}_{\mathrm{HHb}}\left(\lambda_{2}\right)}{\mathcal{E}_{\mathrm{HHb}}\left(\lambda_{1}\right)-\mathcal{E}_{\mathrm{CO}_{2} \mathrm{Hb}}\left(\lambda_{1}\right)-R \cdot\left[\mathcal{E}_{\mathrm{HHb}}\left(\lambda_{2}\right)-\mathcal{E}_{\mathrm{CO}_{2} \mathrm{Hb}}\left(\lambda_{2}\right)\right]}
$$


The feasibility of pulse carbametry will be assessed as follows: considering the extinction coefficients $\mathcal{E}_{\mathrm{CO}_{2} \mathrm{Hb}}$ and $\mathcal{E}_{\mathrm{HHb}}$ derived from our spectrophotometric measurements, and the aforementioned standard deviation on $R$, the standard deviation on $\mathrm{SatCO}_{2}$ will be computed for each $\left(\lambda_{1}, \lambda_{2}\right)$ couple on the 235- to 1000-nm range. Then, finding the minimum value of this deviation with respect to $\left(\lambda_{1}, \lambda_{2}\right)$ will provide the best achievable accuracy using pulse carbametry. More explicitly, the minimum $\mathrm{SatCO}_{2}$ accuracy reachable with pulse carbametry is given as

$$
\delta \mathrm{SatCO}_{2}=\min _{\left(\lambda_{1}, \lambda_{2}\right)} \sigma \operatorname{SatCO}_{2}\left(\lambda_{1}, \lambda_{2}\right)
$$

wherein $\sigma \operatorname{SatCO}_{2}\left(\lambda_{1}, \lambda_{2}\right)$ is the relative standard deviation of $\operatorname{SatCO}_{2}\left(\lambda_{1}, \lambda_{2}\right)$, computed from the $R$ standard deviation in the case of pulse oximetry (5.6\%). Judging whether a given minimal accuracy $\delta \mathrm{SatCO}_{2}$ is small enough is essentially an arbitrary choice. Still, one can rely on the clinically accepted range for $\mathrm{Pa}_{\mathrm{CO}_{2}}$, which is $\pm 7.5 \mathrm{mmHg}$ (95\% limits of agreement, corresponding to \pm 2 S.D.). ${ }^{22}$ Such a range translates into a standard deviation of $9 \%$ on $\mathrm{Pa}_{\mathrm{CO}_{2}}$ reading, at a standard $\mathrm{Pa}_{\mathrm{CO}_{2}}$ level of $40 \mathrm{mmHg}$. Thus, the decision threshold on $\delta \mathrm{SatCO}_{2}$ was set to this value as a first approximation, i.e., if $\delta \mathrm{SatCO}_{2}$ is below $9 \%$, pulse carbametry will be considered feasible.

\section{Results}

\subsection{Hemoglobin Spectra}

The obtained spectra of diluted lysed blood equilibrated with ambient air, pure $\mathrm{N}_{2}$, and pure $\mathrm{CO}_{2}$ are presented in Fig. 3 after baseline correction and outlier removal. Each spectrum is averaged over (UV-Vis/Vis-IR spectra number): 24/13 $\left(\mathrm{CO}_{2}\right), 10 / 17\left(\mathrm{~N}_{2}\right)$, and 32/38 (air) measurements. The variations in the number of trials are explained by several reasons. At first, there were two different measurement campaigns for the UV-Vis and Vis-IR range, leading to less measurements in the Vis-IR (some fluorescence measurements were performed instead, unpublished). Then, there were twice as much measurements made with $\mathrm{O}_{2} \mathrm{Hb}$ as with $\mathrm{N}_{2}$ or $\mathrm{CO}_{2}$, only because $\mathrm{O}_{2} \mathrm{Hb}$ was readily available and measured while waiting for $\mathrm{HHb}$ and $\mathrm{CO}_{2} \mathrm{Hb}$ to be obtained by tonometry. Finally, we used an outlier removal algorithm based on the standard deviation of the measurements, removing measurements diverging more than roughly \pm 2.5 standard deviation from the mean, using an adaptative threshold and a recursive algorithm inspired by the work of Hadi et al. ${ }^{23}$. This threshold choice was arbitrary, as is that of outlier detection and removal in the general case. ${ }^{24}$ Given the important number of wavelengths in each measurement and the limited number of measurements performed, a power calculation was not feasible.

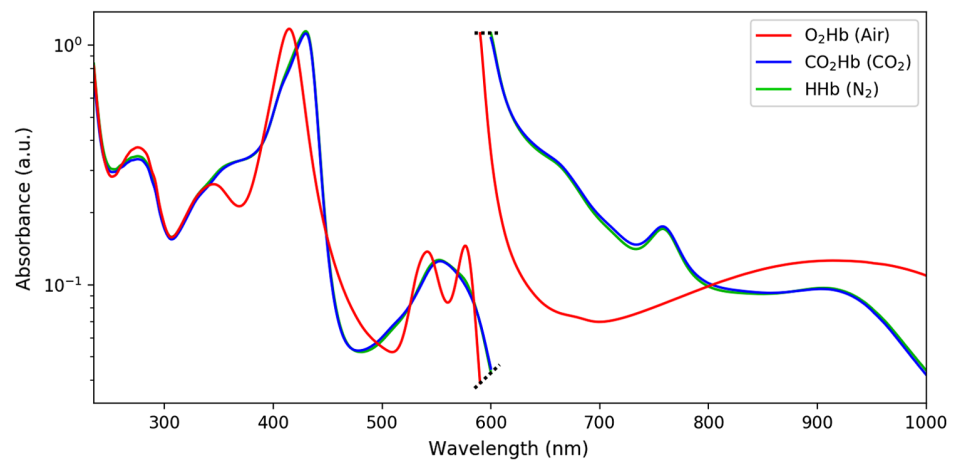

Fig. 3 Measured absorption spectra of diluted lysed blood tonometered with $\mathrm{O}_{2}, \mathrm{~N}_{2}$, or $\mathrm{CO}_{2}$. The vertical scale is arbitrary, data were scaled for representation and corresponds to two different dilution ratios of $1: 1000$ for the UV-Vis 235 to $600 \mathrm{~nm}$ range (left) and 1:10 for the Vis-IR 600- to $1000-\mathrm{nm}$ range (right) (ranges are 235 to $590 \mathrm{~nm}$ and 590 to $1000 \mathrm{~nm}$ for air-equilibrated solutions). The black dashed line separates the UV-Vis from the Vis-IR measurements. 


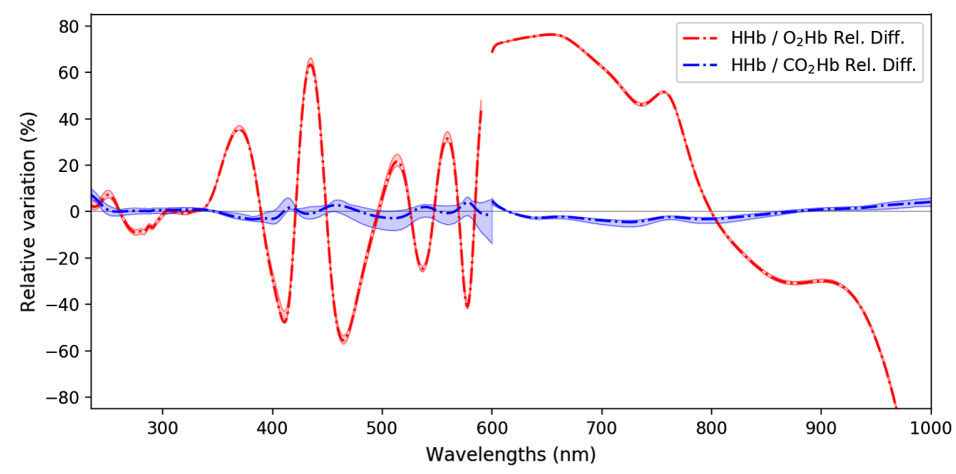

Fig. 4 Relative differences between $\mathrm{HHb}$ and $\mathrm{CO}_{2} \mathrm{Hb}$ (in blue) and between $\mathrm{HHb}$ and $\mathrm{O}_{2} \mathrm{Hb}$ (in red) absorption spectra. The two thin lines for each comparison represent the maximum and minimum values along all measurements. For instance, for $\mathrm{HHb} / \mathrm{CO}_{2} \mathrm{Hb}$ comparison, at a given wavelength, the higher line represents $\mathrm{HHb}$ maximum value minus $\mathrm{CO}_{2} \mathrm{Hb}$ minimum value, and the lower line represents $\mathrm{HHb}$ minimum value minus $\mathrm{CO}_{2} \mathrm{Hb}$ maximum value.

The absorption spectra of $\mathrm{HHb}$ and $\mathrm{CO}_{2} \mathrm{Hb}$ appear to be extremely close, especially compared to $\mathrm{O}_{2} \mathrm{Hb}$. The standard deviations in absorption for $\mathrm{O}_{2} \mathrm{Hb}, \mathrm{CO}_{2} \mathrm{Hb}$, and $\mathrm{HHb}$ were (mean standard deviation, minimum, and maximum) $1.20_{0.38}^{7.55} \mathrm{mAbs}, 3.16_{0.51}^{36.34} \mathrm{mAbs}$, and $2.43_{0.40}^{21.02} \mathrm{mAbs}$, corresponding to relative variations of $0.53_{0.14}^{2.24} \%, 1.08_{0.05}^{5.74} \%$, and $0.81_{0.07}^{4.59} \%$, respectively.

Figure 4 gives a more quantitative analysis to the difference among $\mathrm{O}_{2} \mathrm{Hb}, \mathrm{CO}_{2} \mathrm{Hb}$, and $\mathrm{HHb}$ spectra. While the relative differences between $\mathrm{O}_{2} \mathrm{Hb}$ and $\mathrm{HHb}$ spectra-clearly visible in Fig. 3-reach several tens of percent, those between $\mathrm{CO}_{2} \mathrm{Hb}$ and $\mathrm{HHb}$ are much more tenuous. The differences between mean $\mathrm{O}_{2} \mathrm{Hb}$ and $\mathrm{HHb}$ spectra on the one hand and $\mathrm{CO}_{2} \mathrm{Hb}$ and $\mathrm{HHb}$ spectra on the other hand were (mean absolute difference and maximum) $0.18^{(1.99)}$ Abs and $0.08^{(0.11)}$ Abs, corresponding to relative variations of $36^{(153)} \%$ and $2^{(13)} \%$, respectively.

\subsection{Intra Medium}

Figure 5 shows the spectra of the dilution medium with and without sodium metabisulfite. Results with EDTA are not shown since they were indistinguishable from pure dilution medium on a full scale view. More subtle effects of these substances are shown in Fig. 6, which focuses on the deviation - with respect to pure dilution medium — of the prepared EDTA or sodium metabisulfite solutions. Again, each spectrum is averaged over several measurements. For the UVVis/Vis-IR range: 28/34 (pure) and 12/11 (sodium metabisulfite) measurements. For the Vis-IR only range: five (EDTA) measurements.

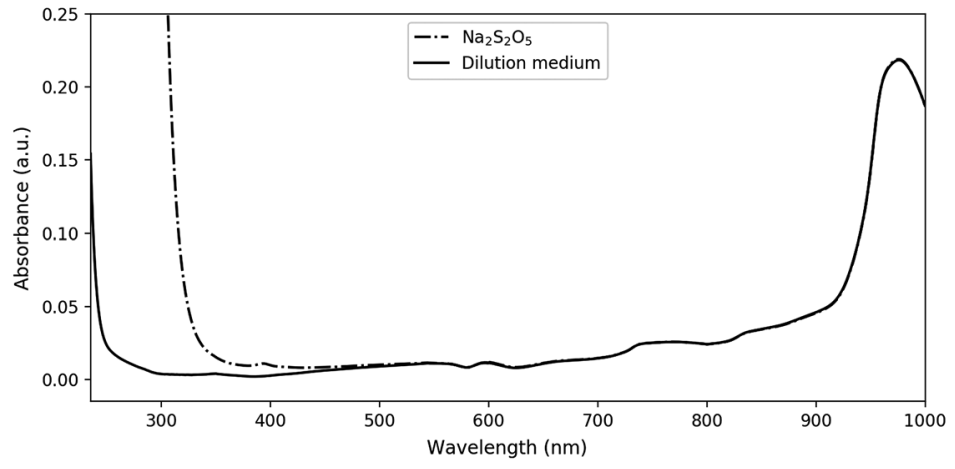

Fig. 5 Absorption spectra of the dilution medium with and without the addition of sodium metabisulfite $(2.0 \mathrm{mM})$, we can observe a marked absorbing effect of sodium metabisulfite in the ultraviolet, up to almost $500 \mathrm{~nm}$. 


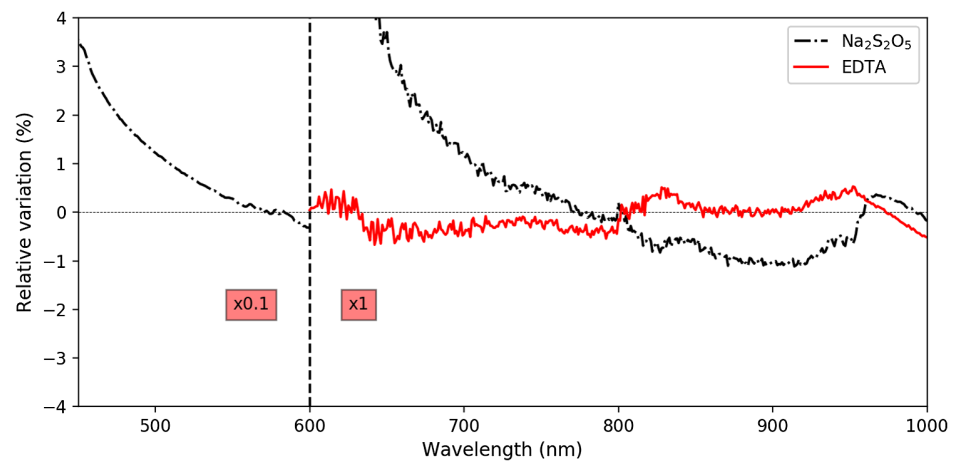

Fig. 6 Relative variations of the dilution medium absorption spectrum upon the addition of EDTA $(0.61 \mathrm{mM})$ or sodium metabisulfite $(2.0 \mathrm{mM})$. The left side is multiplied by $0.1(\sim 10 \%$ of relative variation for sodium metabisulfite at $500 \mathrm{~nm}$ ).

\subsection{Tonometry Duration}

The influence of tonometry duration on the measured absorbances was found to be insignificant for durations between 30 and $45 \mathrm{~min}$, with test results being $\rho=-0.13, p=0.62$, on 17 samples for $\mathrm{N}_{2}$ tonometry and $\rho=-0.04, p=0.91$, on 13 samples for $\mathrm{CO}_{2}$ tonometry (Pearson correlation coefficient). We concluded that $30 \mathrm{~min}$ of equilibration time were enough to obtain either $\mathrm{CO}_{2} \mathrm{Hb}$ or $\mathrm{HHb}$.

\subsection{Pulse Carbametry}

The obtained $\mathrm{CO}_{2} \mathrm{Hb}$ and $\mathrm{HHb}$ spectra- $-\mathrm{CO}_{2}$ and $\mathrm{N}_{2}$ tonometry, respectively-shown in Fig. 3 were then used to perform the analysis presented in Sec. 2.2.3. The value of $\sigma \mathrm{SatCO}_{2}$ as a function of the two wavelengths $\left(\lambda_{1}, \lambda_{2}\right)$ is shown in Fig. 7.

The minimal value taken by $\sigma \mathrm{SatCO}_{2}$ is $\delta \mathrm{SatCO}_{2}=34.2 \%$ at $\lambda_{1}=508 \mathrm{~nm}$ and $\lambda_{2}=$ $600 \mathrm{~nm}$. However, as can be seen from the asymmetry of the spots on the $\lambda_{1}=600 \mathrm{~nm}$ or $\lambda_{2}=$ $600 \mathrm{~nm}$ lines, these values likely originate from limitation of the measurement system. Indeed, with a 1:1000 dilution, lysed blood absorption was below 1.2 Abs on the full 235- to 600-nm range. At the opposite, the 1:10 dilution topped at $2.8 \mathrm{Abs}$ near $600 \mathrm{~nm}$, which is close to the saturation value of $3.0 \mathrm{Abs}$ of the spectrophotometer that we used. Ideally, to circumvent this flaw of the measurement setup, three dilutions at 1:10, 1:100, and 1:1000 could have been performed on the 235 to $450 \mathrm{~nm}, 450$ to $650 \mathrm{~nm}$, and 650 to $1000 \mathrm{~nm}$ ranges, respectively.

Still, even with these flaws, which-in the worst case-could increase the spectral differences between $\mathrm{HHb}$ and $\mathrm{CO}_{2} \mathrm{Hb}$ and thus lower the error on $\mathrm{SatCO}_{2}$ measurements, the reached accuracy is still far above the $9 \%$ target established previously. If $\sigma \mathrm{SatCO}_{2}$ values near

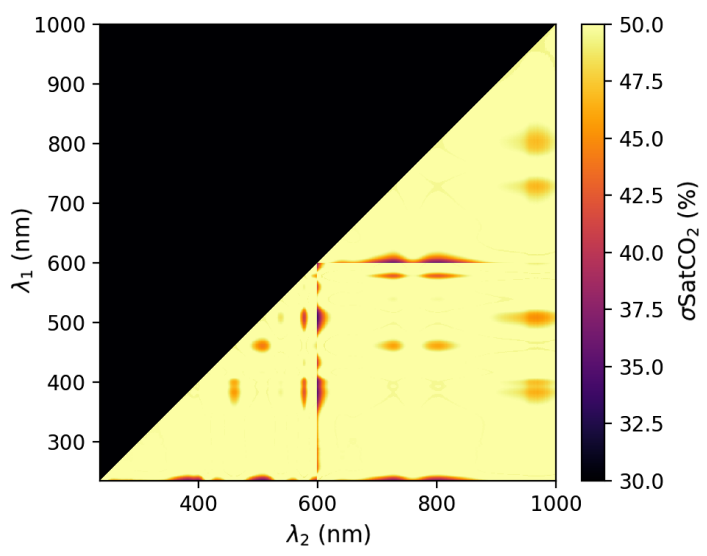

Fig. $7 \sigma \mathrm{SatCO}_{2}$ as a function of $\left(\lambda_{1}, \lambda_{2}\right)$ on the 235- to 1000-nm range. 
the $600-\mathrm{nm}$ lines are discarded and focus is made on other spots such as the $\left(\lambda_{1}=510 \mathrm{~nm}\right.$, $\lambda_{2}=580 \mathrm{~nm}$ ) area, values above $41 \%$ are to be found for $\delta \mathrm{SatCO}_{2}$.

\section{Discussion}

At first, the chosen method for obtaining $\mathrm{CO}_{2} \mathrm{Hb}, \mathrm{HHb}$, and $\mathrm{O}_{2} \mathrm{Hb}$ is discussed. Then, the hemoglobin spectra are compared with those available in the literature, the influence of EDTA and sodium metabisulfite are also discussed. Finally, the feasibility of pulse carbametry is assessed.

\subsection{On the Chosen Method}

Among the several authors who measured hemoglobin absorption spectra, some used freshly drawn blood ${ }^{16,25-33}$ whereas others preferred lyophilized hemoglobin. ${ }^{34-36}$ Lyophilized blood, despite its convenience, has several drawbacks. First, it is composed merely of MetHb ${ }^{33,37}$ and thus needs an oxidation procedure to convert it back into $\mathrm{O}_{2} \mathrm{Hb}$, a step that involves chemicals that might interfere with the hemoglobin spectrum. Second, a better affinity of hemoglobin has been reported for hemoglobin extracted from freshly drawn blood. ${ }^{25,33}$ These considerations drove our choice toward fresh blood as a hemoglobin source.

However, fresh blood requires the lysis of erythrocytes and other blood cells to yield a limpid solution. Despite the common use of a surfactant such as Sterox $\mathrm{SE}^{27,28,32}$ or equivalent, ${ }^{25,29,31}$ we preferred an ultrasound lysis, which adds no foreign chemical in blood for the same effect. ${ }^{30}$

Fresh blood sampling could also require centrifugation to keep only the erythrocytes and avoid spectral interferences from other blood components, namely leucocytes and lipids. Yet, since hemoglobin is the main absorbing compound in blood, by two up to three orders of magnitude ${ }^{38}$ we did not consider the centrifugation step mandatory. Lastly, fresh blood sampling requires the addition of an anticoagulant if it is not largely diluted. Consequently, for the 1:10 dilution ratio- on the 600- to 1000-nm range-we considered the addition of EDTA to the collected blood. Its spectral influence was measured and found to be negligible on the studied range-as shown in Fig. 6-with relative absorption variations in the $\pm 1 \%$ range, corresponding to absolute variations in the $\pm 1 \mathrm{mAbs}$ range, well below the measurement standard deviation $\left(1.24_{-9.3}^{+14.1} \mathrm{mAbs}\right.$ for the dilution medium itself for instance, similar values were found for dilution medium with EDTA). We concluded that EDTA did not have any influence on the measured spectra, in the quantity used in our experiments.

Concerning hemoglobin measurements in its reduced form, the use of sodium dithionite $\left(\mathrm{Na}_{2} \mathrm{~S}_{2} \mathrm{O}_{4}\right)$ has been reported, as a mean to quickly obtain $\mathrm{HHb} \cdot{ }^{26,27,30,33,36}$ Alas, it has also been reported to alter its absorption spectrum. ${ }^{16,35,39}$ We conducted investigations on the possible use of sodium metabisulfite $\left(\mathrm{Na}_{2} \mathrm{~S}_{2} \mathrm{O}_{5}\right)$, which-like sodium dithionite-yields to the production of aqueous bisulfite anion, the strong reducing agent converting $\mathrm{O}_{2} \mathrm{Hb}$ into $\mathrm{HHb}$. Our results-see Figs. 5 and 6-confirm earlier observations and extend them with quantitative measurements on the 235- to 1000-nm range. We would not recommend the use of bisulfite anion for its strong absorption, especially in the short wavelengths up to $500 \mathrm{~nm}$.

Consequently, tonometry was chosen to obtain $\mathrm{HHb}$ and $\mathrm{CO}_{2} \mathrm{Hb}$. Concerning its duration, 30 min was found to be sufficient in Eschweiler spherical glass tonometers filled with $6 \mathrm{~mL}$ of diluted lysed blood as demonstrated in Sec. 3.3. However, one should bear in mind that this duration is strongly dependent on several parameters, such as the shape of the tonometer used for equilibration, its filling level, or the gas flow rate for instance.

The dilution medium was chosen to correspond to an intracellular medium (with high $\mathrm{K}^{+}$ concentration). $\mathrm{pH}$ was also set to an erythrocyte intracellular value of 7.2 since it has been reported to be that of the inner erythrocytes. ${ }^{40,41}$ It has also been reported ${ }^{35,42,43}$ that the $\mathrm{pH}$ has an impact—although relatively small—on the measured hemoglobin spectra. Concerning the choice of HEPES as a buffer, a better preservation of the hemoglobin oxygenation function was reported with HEPES over Tris/Bis-Tris. ${ }^{44}$ Finally, the chosen dilution ratios are justified since hemoglobin has been reported to follow Beer-Lambert law, would it be for extremely diluted or concentrated solutions. ${ }^{34}$ 


\subsection{Hemoglobin Spectra}

The measured spectra of diluted lysed blood, either equilibrated with $\mathrm{N}_{2}$ or ambient air, are extremely close to that of the literature, as can be seen in Fig. 8. This comforts us in the method that we employed and the above-mentioned choices. The small discrepancies observed between our spectra and that of the literature may be explained by a number of methodological differences. For instance, several authors ${ }^{27,28,32}$ used a surfactant such as Sterox SE to perform the erythrocyte lysis, whereas we chose to use ultrasound. The surfactant may have a spectral impact, which - to the best of our knowledge-has never been quantified. Other authors, even when choosing tonometry, added some sodium dithionite before measuring. ${ }^{16}$ Yet, sodium dithionite, such as sodium metabisulfite, is known to have a marked spectral influence. ${ }^{35}$ Zijlstra et al ${ }^{16}$ also mentioned that in case of too long tonometry, supernatant residues sometimes appeared in the diluted blood, the nature of which was not determined. Although we did not observe such behavior, a small turbidity might have been present in their measurements, or ours. Of all the literature spectra shown in Fig. 8, Prahl and Kolyva only offered raw coefficients, whereas Assendelft and Zijlstra detailed their protocol. It is therefore difficult to analyze the potential sources of differences between their spectra or between their spectra and ours.

Overall, the repeatability of the measurements was fairly good, with mean standard deviation of $0.53 \%, 1.08 \%$, and $0.81 \%$, for $\mathrm{O}_{2} \mathrm{Hb}, \mathrm{CO}_{2} \mathrm{Hb}$, and $\mathrm{HHb}$ measurements, respectively, which can be compared to values between $0.8 \%$ and $2.1 \%$ reported by Zijlstra et al. ${ }^{16}$ The rather high maximum standard deviations reported in Sec. 3.1 (2.24\%, 5.74\%, and $4.59 \%$ for $\mathrm{O}_{2} \mathrm{Hb}$, $\mathrm{CO}_{2} \mathrm{Hb}$, and $\mathrm{HHb}$, respectively) are mainly due to the measurement limits of the spectrophotometer near $600 \mathrm{~nm}$, corresponding to either too low ( $\leq 0.05 \mathrm{Abs})$ absorbance below $600 \mathrm{~nm}$ or too high $(\geq 2.5 \mathrm{Abs})$ absorbance above $600 \mathrm{~nm}$. When computing the mean standard deviation without the 580- to 620-nm range for $\mathrm{O}_{2} \mathrm{Hb}, \mathrm{CO}_{2} \mathrm{Hb}$, and $\mathrm{HHb}$, maximum standard deviations values drop to $7.25,12.60$, and $21.02 \mathrm{mAbs}$, corresponding to relative variations of $1.31 \%$, $2.78 \%$, and $2.54 \%$, respectively. The mean absorption spectra that we measured for $\mathrm{O}_{2} \mathrm{Hb}$, $\mathrm{CO}_{2} \mathrm{Hb}$, and $\mathrm{HHb}$ are published as Supplementary Material (see also the supplemental "readme" file explaining the units used).

Fig. 3 also reveals that the spectra of $\mathrm{HHb}$ and $\mathrm{CO}_{2} \mathrm{Hb}$ are extremely close, their differences being more than one order of magnitude below the one between the spectra of $\mathrm{O}_{2} \mathrm{Hb}$ and $\mathrm{HHb}$, as can be seen in Fig 4. Moreover, the measured $\mathrm{HHb}$ and $\mathrm{CO}_{2} \mathrm{Hb}$ spectra are similar to the $\mathrm{HHb}$ spectra already available in the literature, as can be seen in Fig. 8. Such observations tend to make one believe that the formation of carbamined compounds between $\mathrm{CO}_{2}$ and hemoglobin terminal amine groups does not change the hemoglobin molecule conformation significantly, hence bringing no spectral alteration. However, such intuition shall not have the value of evidence, this is why the possible use of slight differences between $\mathrm{HHb}$ and $\mathrm{CO}_{2} \mathrm{Hb}$ will now be discussed.

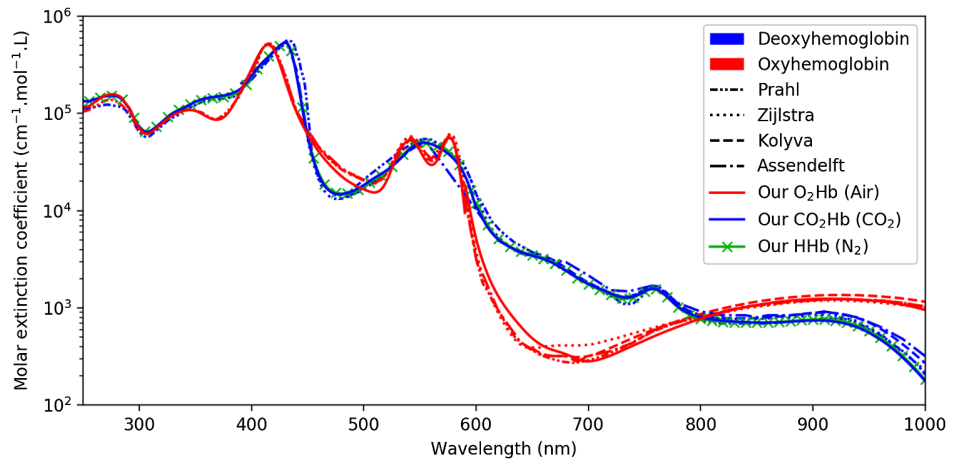

Fig. 8 Our measurements (plain line) compared to that of Prahl, ${ }^{45}$ Zijlstra et al., ${ }^{16}$ Kolyva et al., ${ }^{46}$ and Assendelft. ${ }^{27}$ Our measurements are consistent with that of the literature for $\mathrm{O}_{2} \mathrm{Hb}$ (air) and $\mathrm{HHb}\left(\mathrm{N}_{2}\right)$. 


\subsection{Pulse Carbametry}

Given the accuracy threshold that was fixed at $9 \%$ for $\delta \mathrm{SatCO}_{2}$, and the observed value of $34.2 \%$ - or even more if the values close to the spectrophotometer saturation limit are discarded — we could readily conclude that pulse carbametry — as it was presented — is not feasible. However, several additional aspects of this technique need to be further discussed.

At first sight, the consideration of a binary system composed solely of $\mathrm{HHb}$ and $\mathrm{CO}_{2} \mathrm{Hb}$ can seem surprising. Indeed, in practice, human arterial blood is composed at least of $\mathrm{O}_{2} \mathrm{Hb}, \mathrm{HHb}$, and $\mathrm{CO}_{2} \mathrm{Hb}$ - and even $\mathrm{COHb}^{47,48}$ and $\mathrm{MetHb}^{49,50}$ in small amounts. That being said, it should be clear that the demonstrated inability to distinguish between $\mathrm{CO}_{2} \mathrm{Hb}$ and $\mathrm{HHb}$, even when they are the only absorbing compounds involved, would be worsened by the addition of any other perturbing absorbing species- e.g., $\mathrm{O}_{2} \mathrm{Hb}$. An in-depth analysis of the tertiary system $\mathrm{O}_{2} \mathrm{Hb}-$ $\mathrm{HHb}-\mathrm{CO}_{2} \mathrm{Hb}$ would have been necessary only if pulse carbametry had been found to be possible in a binary system.

Then, we made the hypothesis that all studied errors were Gaussian. It is most often considered to be the case in the literature, ${ }^{5,6}$ and we will also stick to this hypothesis in the absence of evidence to the contrary. Thus, the main remaining question is to know whether the mathematical functions giving $R=f\left(\mathrm{SatO}_{2}\right)$ and $\mathrm{SatCO}_{2}=g(R)$ can be reasonably linearly approximated. The latter assumption has to be checked for $f$ at the $660 / 940 \mathrm{~nm}$ couple, and for $g$ on the full 235 to $1000 \mathrm{~nm}$ range. The calculation of the derivative of these two functions is straightforward and allows one to conclude that the relative variation of the slope of $f$ stays below $3.8 \%$ on the 70 - to $100 \%-\mathrm{SatO}_{2}$ range at $660 / 940 \mathrm{~nm}$, whereas the slope of $g$ stays below $2.2 \%$ on the $0 \%$ to $100 \%$ $\mathrm{SatCO}_{2}$ range at $340 / 600 \mathrm{~nm}$ (maximal value on the 235- to 1000-nm range). We can thus safely conclude that approximating $f$ and $g$ with linear functions is reasonable and that our hypothesis concerning Gaussian errors is justified.

Next, all the calculations leading to Fig. 7 were made considering a monochromatic skin illumination. In a typical pulse carbametry application, the light source is more likely to be a laser, laser diode, or light-emitting diode (LED). In such cases, the effect of a nonmonochromatic light source will be a degradation of $\delta \mathrm{SatCO}_{2}$ caused by the spectral spread of the source. Such spread will basically smooth $\mathrm{HHb}$ and $\mathrm{CO}_{2} \mathrm{Hb}$ spectra by convolving them with the emission spectrum of the source. Such spectrum can in turn be roughly regarded as a Gaussian window of full-width at half-maximum (FWHM) of a few (laser sources) or a few tenths (LED) of nanometers. For instance, a source with an FWHM of $5 \mathrm{~nm}$ leads to a $\delta \mathrm{SatCO}_{2}$ of $36.8 \%$, with an FWHM of $20 \mathrm{~nm}$ this value reaches $42.1 \%$, to be compared with the $34.2 \%$ of the aforementioned ideal monochromatic case.

Finally, the last assumption that we made concerns the extrapolation to pulse carbametry of the accuracy on $R$ measurement in the $\mathrm{SatO}_{2}$ case. Such an assumption was made considering that the 660- and 940-nm wavelengths were not chosen randomly but to maximize pulse oximetry sensitivity. In other words, they were chosen such that a small change in $\mathrm{SatO}_{2}$ translates into a huge change in measured light intensity at certain wavelengths, ${ }^{27,51}$ but also such that they were in the tissues optical window-the 700 - to 1000 -nm range. ${ }^{52-54}$ Such considerations make the $\mathrm{SatO}_{2}$ $660 / 940 \mathrm{~nm}$ situation a best case, and we would expect other wavelengths couples to give equally or worse accurate $R$ measurements. This remains, however, a supposition since-to the best of our knowledge - there appears to be no study on the measurement accuracy on $\mathrm{SatO}_{2}$ - and thus $R$ at wavelengths different from the usual $660 / 940 \mathrm{~nm}$ pair. Still, it is worth noticing that our conclusions would remain unchanged, even if we managed somehow to drastically reduce the measuring accuracy on $R$ - say by a factor two or three, we would still have a $\delta \mathrm{SatCO}_{2}$ value above $9 \%$.

\section{Conclusion}

In this study, $\mathrm{CO}_{2} \mathrm{Hb}$ absorption spectrum was measured for the first time. $\mathrm{O}_{2} \mathrm{Hb}$, $\mathrm{HHb}$, and $\mathrm{CO}_{2} \mathrm{Hb}$ were obtained from diluted lysed blood equilibrated with ambient air, pure $\mathrm{N}_{2}$, and pure $\mathrm{CO}_{2}$, respectively. Their isolation method was discussed thoroughly, including the possible use of EDTA or sodium metabisulfite.

The absorption spectra of $\mathrm{O}_{2} \mathrm{Hb}$ and $\mathrm{HHb}$ were close to that of literature, whereas the absorption spectrum of $\mathrm{CO}_{2} \mathrm{Hb}$ was extremely close to that of $\mathrm{HHb}$. No influence of EDTA was found. 
Sodium metabisulfite, however, strongly absorbs in the ultraviolet and visible range up to $500 \mathrm{~nm}$. As such, it should not be used in this spectral range for hemoglobin reduction.

A theoretical variation of pulse oximetry applied to the determination of $\mathrm{CO}_{2} \mathrm{Hb}$ fraction was presented, called pulse carbametry. Such theory was applied to the aforementioned measurements to conclude whether the slight variations observed between $\mathrm{HHb}$ and $\mathrm{CO}_{2} \mathrm{Hb}$ absorption spectra could be used in such context. Our observations show that such approach seems extremely challenging since these spectra are almost identical. In particular, on the basis of current knowledge, pulse carbametry may not be used in medical practice.

Yet, this work may benefit from further investigations to consolidate its conclusions. In particular, only two wavelengths were considered for pulse carbametry. It would be interesting to use more sophisticated multiwavelengths approaches since they usually give better results in the case of pulse oximetry ${ }^{20}$ although the question of wavelengths selection becomes more complex. ${ }^{55,56}$

\section{Disclosures}

The authors declare that there are no conflicts of interest related to this article.

\section{Acknowledgments}

We would like to express our special thanks to Karine Pichavant for her technical and scientific support during the measurements, Céline Théron for her technical help, and Sylvain Gioux for his redacting advices. This work was funded by BiOSENCY and the Universite de Bretagne Occidentale. Some of the graphic symbols presented in Fig. 1 were provided by Icon Island, Eucalyp, Teresa Moravcova, Adrien Coquet, Turkubb, and Amrit Mazumder from the Noun Project and are under Creative Comons license (CC BY 3.0).

\section{References}

1. P. D. Wagner, "The physiological basis of pulmonary gas exchange: implications for clinical interpretation of arterial blood gases," Eur. Respir. J. 45(1), 227-243 (2015).

2. T. Bradley et al., "Daytime hypercapnia in the development of nocturnal hypoxemia in COPD," Chest 97, 308-312 (1990).

3. E. Kerem et al., "Prediction of mortality in patients with cystic fibrosis," New Engl. J. Med. 326(18), 1187-1191 (1992).

4. A. M. Al Rajeh and J. R. Hurst, "Monitoring of physiological parameters to predict exacerbations of chronic obstructive pulmonary disease (COPD): a systematic review," J. Clin. Med. 5, 108 (2016).

5. M. Nitzan, A. Romem, and R. Koppel, "Pulse oximetry: fundamentals and technology update," Med. devices: Evidence Res. 7, 231-239 (2014).

6. A. Jubran, "Pulse oximetry," Crit. Care 19, 272 (2015).

7. T. A. Raffin, "Diagnostic decision: indications for arterial blood gas analysis," Ann. Internal Med. 105(3), 390-398 (1986).

8. B. V. Scheer, A. Perel, and U. J. Pfeiffer, "Clinical review: complications and risk factors of peripheral arterial catheters used for haemodynamic monitoring in anaesthesia and intensive care medicine," Crit. Care 6, 199 (2002).

9. A. A. Nanji and K. J. Whitlow, "Is it necessary to transport arterial blood samples on ice for pH and gas analysis?" Can. Anaesth. Soc. J. 31 (1984).

10. S. D. McSwain et al., "End-tidal and arterial carbon dioxide measurements correlate across all levels of physiologic dead space," Respir. Care 55(3), 288-293 (2010).

11. M. E. Donnellan, "Capnography: gradient $\mathrm{PaCo}_{2}$ and $\mathrm{PetCo}_{2}$," Appl. Technol. Pulmonary Med. 126-131 (2011).

12. J. W. Severinghaus and P. B. Astrup, "History of blood gas analysis. III. Carbon dioxide tension," J. Clin. Monitor. 2, 60-73 (1986). 
13. A. Conway et al., "Accuracy and precision of transcutaneous carbon dioxide monitoring: a systematic review and meta-analysis," Thorax 74, 157-163 (2018).

14. C. Geers and G. Gros, "Carbon dioxide transport and carbonic anhydrase in blood and muscle," Physiol. Rev. 80, 681-715 (2000).

15. A. Lumb, Nunn's Applied Respiratory Physiology, 8th ed., Elsevier (2016).

16. W. G. Zijlstra, A. Buursma, and O. W. van Assendelft, Visible and Near Infrared Absorption Spectra of Human and Animal Hemoglobin: Determination and Application, VSP (2000).

17. A. C. Rencher, Methods of Multivariate Analysis, 2nd ed., John Wiley and Sons (2002).

18. G. A. Millikan, "The oximeter, an instrument for measuring continuously the oxygen saturation of arterial blood in man," Rev. Sci. Instrum. 13(10), 434-444 (1942).

19. A. Zwart et al., "Multicomponent analysis of hemoglobin derivatives with reversed-optics spectrophotometer," Clin. Chem. 30(3), 373-379 (1984).

20. K. Urpalainen, "Development of a fractional multi-wavelength pulse oximetry algorithm," Master's Thesis, Aalto University (2011).

21. T. Tamura et al., "Wearable photoplethysmographic sensors-past and present," Electronics 3(2), 282-302 (2014).

22. K. Bendjelid et al., "Transcutaneous $\mathrm{Pco}_{2}$ monitoring in critically ill adults: clinical evaluation of a new sensor," Crit. Care Med. 33, 2203-2206 (2005).

23. A. Hadi, "Identifying multiple outliers in multivariate data," J. R. Stat. Soc. Ser. B (Methodol.) 54 (1992).

24. H. Aguinis, R. K. Gottfredson, and H. Joo, "Best-practice recommendations for defining, identifying, and handling outliers," Organ. Res. Methods 16(2), 270-301 (2013).

25. B. L. Horecker, "The absorption spectra of hemoglobin and its derivatives in the visible and near infra-red regions," J. Biol. Chem. 148(1), 173-183 (1943).

26. R. B. Barlow and M. L. Polanyi, "Absorption measurements for oxygenated and reduced hemoglobin in the range 0.6-1.88 microns," Clin. Chem. 8(1), 67-71 (1962).

27. O. W. V. Assendelft, Spectrophotometry of Haemoglobin Derivatives, Van Gorcum, Assen (Summary in Spanish) (1970).

28. G. A. Mook et al., "Spectrophotometric determination of oxygen saturation of blood independent of the presence of indocyanine green," Cardiovasc. Res. 13, 233-237 (1979).

29. S. Wray et al., "Characterization of the near infrared absorption spectra of cytochrome aa3 and haemoglobin for the non-invasive monitoring of cerebral oxygenation," Biochim. Biophys. Acta - Bioenergetics 933(1), 184-192 (1988).

30. Y. Mendelson and J. C. Kent, "Variations in optical absorption spectra of adult and fetal haemoglobins and its effect on pulse oximetry," IEEE Trans. Biomed. Eng. 36, 844-848 (1989).

31. M. Cope, "The application of near infrared spectroscopy to non-invasive monitoring of cerebral oxygenation in the newborn infant, PhD Thesis, University of London (1991).

32. W. Zijlstra and W. Meeuwsenvanderroest, "Absorption spectra of human, fetal and adult oxyhemoglobin, de-oxyhemoglobin, carboxyhemoglobin, and methemoglobin," Clin. Chem. 37, 1633-1638 (1991).

33. E. Mieczkowska, R. Koncki, and L. Tymecki, "Hemoglobin determination with paired emitter detector diode," Anal. Bioanal. Chem. 399, 3293-3297 (2010).

34. D. L. Drabkin and J. H. Austin, "Spectrophotometric studies: V. A technique for the analysis of undiluted blood and concentrated hemoglobin solutions," J. Biol. Chem. 112(1), 105-115 (1935).

35. K. A. Dalziel and J. P. O'brien, "Side reactions in the deoxygenation of dilute oxyhaemoglobin solutions by sodium dithionite," Biochem. J. 67(1), 119-124 (1957).

36. F. E. Robles, S. Chowdhury, and A. Wax, "Assessing hemoglobin concentration using spectroscopic optical coherence tomography for feasibility of tissue diagnostics," Biomed. Opt. Express 1, 310-317 (2010).

37. Sigma-Aldrich, "Human haemoglobin lyophilised powder, H7379, product page," (2020).

38. M. C. Meinke et al., "Optical properties of platelets and blood plasma and their influence on the optical behavior of whole blood in the visible to near infrared wavelength range," J. Biomed. Opt. 12(1), 014024 (2007). 
39. G. Schubart et al., "Spektralphotometrie von hämolysiertem blut im nahen infrarot," Pflüger's Archiv für die gesamte Physiologie des Menschen und der Tiere 265, 1-10 (1957).

40. F. B. Jensen, "Red blood cell $\mathrm{pH}$, the Bohr effect, and other oxygenation-linked phenomena in blood $\mathrm{O}_{2}$ and $\mathrm{Co}_{2}$ transport," Acta Phys. Scand. 182(3), 215-227 (2004).

41. D. Kummerow et al., "Variations of intracellular $\mathrm{pH}$ in human erythrocytes via $\mathrm{K}^{+}\left(\mathrm{Na}^{+}\right) /$ $\mathrm{H}^{+}$exchange under low ionic strength conditions," J. Membr. Biol. 176, 207-216 (2000).

42. P. D. Wimberley et al., "Effect of $\mathrm{pH}$ on the absorption spectrum of human oxyhemoglobin: a potential source of error in measuring the oxygen saturation of hemoglobin," Clin. Chem. 34(4), 750-754 (1988).

43. W. Zijlstra and A. Buursma, "Spectrophotometry of hemoglobin: absorption spectra of bovine oxyhemoglobin, deoxyhemoglobin, carboxyhemoglobin, and methemoglobin," Comp. Biochem. Physiol. Part B: Biochem. Mol. Biol. 118(4), 743-749 (1997).

44. R. Weber, "Use of ionic and zwitterionic (Tris/BisTris and HEPES) buffers in studies on hemoglobin function," J. Appl. Physiol. (Bethesda, Md.: 1985) 72, 1611-1615 (1992).

45. S. Prahl, "Tabulated molar extinction coefficient for hemoglobin in water," (1998).

46. C. Kolyva et al., "Systematic investigation of changes in oxidized cerebral cytochrome c oxidase concentration during frontal lobe activation in healthy adults," Biomed. Opt. Express 3, 2550-2566 (2012).

47. P. M. Mcllvaine, W. C. Nelson, and D. Bartlet, "Temporal variation of carboxyhemoglobin concentrations," Arch. Environ. Health: Int. J. 19(1), 83-91 (1969).

48. N. J. Wald et al., "Carbon monoxide in breath in relation to smoking and carboxyhaemoglobin levels," Thorax 36(5), 366-369 (1981).

49. H. Kravitz et al., "Methemoglobin values in premature and mature infants and children," AMA J. Diseases Child. 91, 1-5 (1956).

50. E. van Kampen and W. Zijlstra, "Determination of hemoglobin and its derivatives," in Advances in Clinical Chemistry, H. Sobotka and C. Stewart, Eds., Vol. 8, 141-187, Elsevier (1966).

51. G. Mook, O. van Assexdelft, and W. Zijlstra, "Wavelength dependency of the spectrophotometric determination of blood oxygen saturation," Clin. Chim. Acta 26(1), 170-173 (1969).

52. B. C. Wilson, W. P. Jeeves, and D. M. Lowe, "In vivo and post mortem measurements of the attenuation spectra of light in mammalian tissues," Photochem. Photobiol. 42(2), 153-162 (1985).

53. C. Melo et al., "Characterization of light penetration in rat tissues," J. Clin. Laser Med. Surg. 19, 175-179 (2001).

54. C. Ash et al., "Effect of wavelength and beam width on penetration in light-tissue interaction using computational methods," Lasers Med. Sci. 32, 1909-1918 (2017).

55. J. A. Brunelle et al., "Simultaneous measurement of total hemoglobin and its derivatives in blood using co-oximeters: analytical principles; their application in selecting analytical wavelengths and reference methods; a comparison of the results of the choices made," Scand. J. Clin. Lab. Investig. Suppl. 224, 47-69 (1996).

56. B. J. Brendel and T. Nielsen, "Selection of optimal wavelengths for spectral reconstruction in diffuse optical tomography," J. Biomed. Opt. 14(3), 034041 (2009).

Emmanuel Dervieux is a $\mathrm{PhD}$ student with a background in physics, signal processing, and telecommunications. He reoriented toward applied physiology in his PhD course. His research interest focuses on the chemical and optical properties of human skin and tissues on the one hand, and on transcutaneous sensing techniques on the other hand, aiming at discovering novel, non-invasive schemes for transcutaneous blood gas analysis.

Quentin Bodinier is an engineer from 2014. He received his $\mathrm{PhD}$ in digital systems and signal processing in 2017. He applied his expertise in developing 5G wireless communication systems. He now leads the research and development of innovative biophysical sensors at BiOSENCY, a French SME which focuses on facilitating the remote monitoring of patients with chronic respiratory diseases. There, they focus on two main research areas: (1) development of clinicalgrade and non-invasive biophysical sensors and (2) preventive clinical data analysis. 
Wilfried Uhring received his MSc degree in microelectronic, his master's degree in engineering physic in 1999, and his PhD in optoelectronics from the University of Strasbourg, France, in 2002. His research activity is about high-speed imaging. He designed several ultrafast optical detection devices, such as streak cameras, gated intensified cameras, and fast image CMOS sensors with sub-nanosecond to picosecond resolution for biomedical imaging. He is the author/ coauthor of more than 120 peer-reviewed articles in international journals and conferences.

Michaël Théron is an assistant professor at the University of Brest, France. He is a member of the Laboratory of Physiology ORPHY. His scientific expertise belongs to the fields of animal physiology (mammals and fish), adaptations to physical and chemical constraints, gas transport, bioenergetics, hydrostatic, and gas pressures, with two main research fields: (1) ecotoxicology of fuel oil contamination and assessment of depollution methods and (2) effects of gas and hydrostatic pressures in the context of human diving. 